\title{
Das Offene Technologielabor-The Great Good Place der Kommunalentwicklung?
}

\author{
Helene Schabasser ${ }^{1} \cdot$ Tatjana Fischer $^{2}$ iD $\cdot$ Gernot Stöglehner $^{2}$ iD \\ Eingegangen: 23. Januar 2021 / Überarbeitet: 1. September 2021 / Angenommen: 2. September 2021 / Online publiziert: 16. September 2021 \\ (c) Der/die Autor(en) 2021
}

\section{Zusammenfassung}

Das Offene Technologielabor (Otelo) ist ein themenoffenes Treffpunktkonzept, welches gemäß dem Konzept nach Oldenburg (1989) den Dritten Orten zugeordnet werden kann. Die Besonderheit des Otelo-Konzepts liegt darin, dass es die Förderung von Innovation und Kreativwirtschaft in ländlichen Gemeinden und deren zukunftsfähige wirtschaftliche und soziale Entwicklung zum Ziel hat. Otelo wird seit 2010 auf Vereinsbasis und vorwiegend in Österreich umgesetzt. Bisher wurde jedoch noch nicht empirisch erfasst, welche Rolle die Offenen Technologielabore - und somit der Einsatz des Konzepts der Dritten Orte - für die kommunale Entwicklung einnehmen können und in welchem räumlichen Kontext Otelo-Aktivitäten eingebettet sind. Die vorliegende Betrachtung zu 2 Zeitpunkten (Januar 2016 und September 2019) zeigt, dass Otelo sich vor allem zur Erweiterung des kommunalen Kultur- und Erholungsangebots, zur Standortaufwertung sowie zur Förderung von Initiativen, welche einem auf Wachstum fokussierten Wirtschaftssystem Alternativen einzubringen versuchen, eignet. In seiner Umsetzung hat sich gezeigt, dass sich Otelo vor allem in urbanen und regionalen Zentren sowie ländlichen Gemeinden im Umland von Zentren bewährt. Während Dritte Orte bisher vor allem im Kontext der Privatwirtschaft beschrieben wurden, bietet dieser Beitrag Orientierung zur Eignung und zu den Limitationen der Anwendung dieses Konzepts in der Kommunalentwicklung.

Schlüsselwörter Dritter Ort · Kommunale Entwicklung · Zivilgesellschaftliches Engagement · Soziale Innovation · Daseinsvorsorge

\begin{abstract}
The Open Technology Lab (Otelo) is an open-ended meeting place concept that can be classified as a Third Place according to Oldenburg's (1989) concept. The special feature of the Otelo concept is that it is aimed at promoting innovation and creative industries in rural communities and their sustainable economic and social development. Otelo has been implemented on a voluntary basis since 2010, predominantly in Austria. So far, however, it has not been empirically recorded which role Open Technology Labs - and therefore the concept of Third Places_-play in municipal development and in which spatial context Otelo activities are embedded. The present analysis at two points in time (January 2016 and September 2019) shows that Otelo is suitable for expanding municipal socio-cultural and recreational offerings, for upgrading municipal sites and for promoting initiatives that seek to provide alternatives to an economic system focused on growth. In its implementation, Otelo has proven to be particularly effective in urban and regional centres and rural municipalities in areas surrounding these centres. Although Third Places have so far been described mainly in the context of the private sector, this article offers guidance on the suitability and limitations of applying this concept in municipal development.
\end{abstract}

Keywords Third Places $\cdot$ Municipal development $\cdot$ Civil society engagement $\cdot$ Social innovation $\cdot$ Services of general interest

Mag. Dr. Tatjana Fischer

tatjana.fischer@boku.ac.at

1 Danube Strategy Point, EU-Förderagentur, Kirchberggasse 33-35/9, 1070 Wien, Österreich
2 Institut für Raumplanung, Umweltplanung und Bodenordnung, Universität für Bodenkultur Wien, Peter Jordan-Straße 82, 1190 Wien, Österreich 


\section{Einleitung}

Die im April 2020 erschienene Standort-Ausgabe thematisierte die Relevanz von sogenannten Dritten Orten als wichtige Treffpunkte für den Aufbau sozialer Beziehungen im Kontext der Kreativwirtschaft: So besteht ein Mehrwert von sogenannten Open Creative Labs aus ihrem institutionellen und wirtschaftlichen Umfeld sowie den sozialen und wirtschaftlichen Beziehungen, die sich in diesem Umfeld entwickeln können (Schmidt 2020). Regionale Community Gatherings wie die sogenannten CreativeMornings für Kultur- und Kreativschaffende in Berlin bieten die Möglichkeit zum Knüpfen neuer schwacher Verbindungen und fördern den Faktor „Zufall“ im Austausch von Erfahrungsund Branchenwissen (Müller und Schneider 2020). Auch Co-Working-Spaces folgen teils den Prinzipien Dritter Orte, indem sie auf Gemeinschaft und die Kombination verschiedener Ressourcen bauen und als Treffpunkt und Kommunikationsplattform dienen (Growe et al. 2020). Das Konzept des Dritten Ortes, welches diesen Veranstaltungen und Räumen zugrunde liegt, geht auf Ray Oldenburg und sein 1989 erstmals veröffentlichtes Buch The Great Good Place zurück. Darin definiert er als „Ersten Ort“ das Zuhause und als „Zweiten Ort“ den Arbeitsplatz. „Dritte Orte“ sind Orte der Gemeinschaft, der Interaktion und der Kreativität außerhalb des Zuhauses und des Arbeitsplatzes (Oldenburg 1999, S. 16). Das Konzept der Dritten Orte entwickelt Oldenburg im Kontext der Suburbanisierung der USA. Er argumentiert, dass die Strukturierung nordamerikanischer Vorstädte keinen Raum für Gemeinschaftsleben bieten und zu Einsamkeit und Isolation führen würde. Den Mehrwert Dritter Orte sieht er auf persönlicher wie auf gesellschaftlicher Ebene:

Auf persönlicher Ebene können Dritte Orte eine Alternative zu den Routinen der Ersten und Zweiten Orte bieten. Der Reiz des Neuen wird durch ungeplante Begegnungen und eine lose Struktur gefördert. Die Begegnung mit Menschen außerhalb des bewusst gewählten Freundeskreises kann den persönlichen Horizont erweitern. Dritte Orte dienen außerdem dazu, die Verpflichtungen des Alltags beiseitezulassen, und können in diesem Sinne ein persönliches Druckventil sein. Schließlich können durch die Zugehörigkeit zu einer Gruppe mehrere lose, unverbindliche Freundschaften gleichzeitig gepflegt werden, ohne individuell einen „Beziehungsaufwand“ zu betreiben - der gemeinsame Genuss der Freizeit steht im Vordergrund (Oldenburg 1999, S. 43-65.).

Auf gesellschaftlicher Ebene sieht Oldenburg (1999, S. 66-88) den Mehrwert Dritter Orte im Raum zur Stärkung des gesellschaftlichen Gefüges (,social fabric“) im Sinne der niederschwelligen Teilhabe an politischen Prozessen: Dritte Orte sind Orte des Austauschs von Neuigkeiten und Orte der individuellen sowie kollektiven Meinungsbildung.
Zudem sind sie Orte, an denen Menschen mit ähnlichen Interessen zueinanderfinden können, wodurch in Folge formellere Vereinigungen entstehen können. Sie können Orte der kritischen Reflexion von Inhalten sein, welche über Massenmedien eventuell unkritisch aufgenommen werden. Oldenburg argumentiert weiter, dass Dritte Orte bis zu einem gewissen Grad die in einer individualisierten Gesellschaft nicht mehr wahrgenommenen kollektiven Feste und Feiertage ersetzen können, weil sie eine Atmosphäre der Ungezwungenheit und Ausgelassenheit bieten und eine vereinigende und integrierende Funktion haben. Schließlich sind Dritte Orte auch Räume der gegenseitigen Beobachtung, in denen unerwünschte soziale Normen sanktioniert werden können.

Die Kerncharakteristika Dritter Orte fasst Oldenburg (1999, S. 22-42) in den folgenden 8 Punkten zusammen:

1. Neutrales Umfeld: Dritte Orte sind neutrale Orte, an denen Menschen nach Belieben kommen und gehen können, ohne Verpflichtungen gegenüber anderen Teilnehmer*innen einzugehen. Es sind Orte, die Menschen zusammenbringen, die sich sonst nicht getroffen hätten.

2. Begegnungsort auf Augenhöhe: Dritte Orte sind Räume, in denen der Status einer Person am Arbeitsplatz oder in der Gesellschaft keine Rolle spielt. Die Akzeptanz und Teilnahme an Dritten Orten sind nicht mit Voraussetzungen, Rollen, Pflichten oder dem Nachweis einer bestimmten Zugehörigkeit verbunden.

3. Konversation im Mittelpunkt: An Dritten Orten steht Konversation im Mittelpunkt, wobei ein spielerischer und gewitzter Zugang geschätzt wird.

4. Gute Erreichbarkeit und Zugänglichkeit: Dritte Orte sind gut erreichbar und auch zu Tageszeiten zugänglich, die außerhalb der regulären Arbeits- oder Schulzeiten liegen.

5. Vorhandenes Stammklientel: Dritte Orte zeichnen sich durch Stammgäste aus, die Neuankömmlinge anziehen und dem Ort seine charakteristische Stimmung verleihen.

6. Einfaches, bescheidenes Erscheinungsbild: An Dritten Orten zählt Funktionalität mehr als das Erscheinungsbild. Die Räumlichkeiten sollen der Interaktion und dem Dialog dienen.

7. Spielerische Stimmung: Dritte Orte zeichnen sich durch eine spielerische Stimmung und durch Freude an wortgewandter Interaktion aus. Diese spielerische Stimmung soll dazu beitragen, dass Teilnehmer*innen immer wieder kommen.

8. Ein Zuhause außerhalb von Zuhause: Dritte Orte stellen insofern ein zweites Zuhause dar, da sich Teilnehmer*innen hier entspannen und in einem sozialen Umfeld erholen können, ihrem eigenen Ausdruck freien Lauf lassen können, sich gegenseitig unterstützen und Anteil nehmen. 
Der 2010 in Österreich gegründete Verein Offenes Technologielabor (kurz Otelo) spiegelt diese 8 Kerncharakteristika Dritter Orte gänzlich wider. Zudem erhebt das Offene Technologielabor den Anspruch, „Innovationsräume zu schaffen, die gezielt verschiedene Interessens- und Altersgruppen ansprechen" (Hollinetz et al. 2012, S. 12) und so einen Beitrag zur regionalen Entwicklung zu leisten: „Nicht nur der Erhalt der Lebensqualität steht im Vordergrund, sondern auch die zukunftsfähige wirtschaftliche Entwicklung ländlicher Gebiete“ (Hollinetz et al. 2012, S. 12). Die 2 Alleinstellungsmerkmale des Vereins sind Themenoffenheit und Zieloffenheit: Das Offene Technologielabor versucht ein Umfeld für motivierte Menschen zu bieten, in dem sie ihre Ideen gemeinsam entwickeln und umsetzen können, mit dem Ziel, individuellen sowie kollektiven Mehrwert für die Region zu schaffen. Durch die Initiierung lokaler und regionaler Lernprozesse sollen Wissenstransfer und Innovation sowie die Förderung der Kreativwirtschaft unterstützt werden. Das Offene Technologielabor greift somit drängende Fragen und Bedürfnisse vieler ländlicher wie auch städtischer Gemeinden in Bezug auf Arbeit und Bildung auf. Kern des Otelo-Konzepts sind seitens der Gemeinde kostenlos zur Verfügung gestellte Räumlichkeiten sowie Internet.

Aus dieser Verwendung des Konzepts der Dritten Orte für den Zweck der räumlichen Entwicklung ergibt sich ein Spannungsfeld zwischen Ungezwungenheit und Ungebundenheit auf der einen Seite sowie Zweckgebundenheit und Zielorientierung in Richtung räumliche Entwicklung auf der anderen Seite. Ziel des vorliegenden Beitrags ist es, erstens zu beschreiben, inwieweit die Aktivitäten der Offenen Technologielabore diesem Spannungsfeld standhalten und zur kommunalen Entwicklung beitragen. Zweitens wird geklärt, inwiefern das Konzept seinem Anspruch, Innovation und Kreativwirtschaft in ländlichen Räumen zu fördern, in der Praxis gerecht wird, indem die erfassten Otelo-Gruppen hinsichtlich ihrer räumlichen Dimension (urban - suburban - ländlich), ihrer (sozialen) Innovationsträchtigkeit, ihres Beitrags zur Wirtschaft bzw. Kreativwirtschaft und ihrer Beständigkeit beleuchtet werden. Da der Fokus dieser Analyse auf den Otelo-Standortgemeinden liegt, beschränken sich die Forschungsfragen auf die örtliche Entwicklung.

\section{Methoden}

Zur Beantwortung der Forschungsfragen wird zuerst anhand des Otelo-Handlungsbuchs (Hollinetz et al. 2012) gezeigt, wie das Konzept der Offenen Technologielabore die 8 Kerncharakteristika Dritter Orte widerspiegelt. Zudem werden der räumliche Kontext und die Herausforderungen der betreffenden Standortgemeinden aus Sicht der Bürgermeister*innen der untersuchten Otelo-Standorte dargestellt. Anschließend werden die gegründeten Gruppen per Januar
2016 an den 9 ersten Otelo-Standorten den Daseinsgrundfunktionen der Münchner Schule der Sozialgeografie (Werlen 2000, S. 175-177) zugeordnet, um deren Funktion für die Daseinsvorsorge abbilden zu können. Der Vergleich dieser Gruppen mit den aktiven Gruppen im September 2019 gibt Aufschluss über die Beständigkeit der Otelo-Aktivitäten. Die Gegenüberstellung der Perspektiven von Standortsprecher*innen und Bürgermeister*innen auf Otelo ermöglicht eine konsolidierte Darstellung des Beitrags von Otelo zur kommunalen Entwicklung.

Um die Bedeutung und den Mehrwert von Otelo als Dritten Ort für die Zivilgesellschaft und kommunale Entwicklung in Österreich darzustellen, wurde ein Ansatz gewählt, der auch die „Zeit“ als wichtige Determinante für die Ableitung des „Erfolges“ miteinschließt. Zu diesem Zweck wurden die Aktivitäten der Otelos zu 2 Zeitpunkten, namentlich 2016 und 2019, vergleichend betrachtet - diese kurze Zeitspanne ist dem jungen Alter der Umsetzung dieses Konzepts in Österreich geschuldet - und vor dem Hintergrund der Anforderung als Innovation als Dritte Orte diskutiert.

Den Ausgangspunkt hierfür bildeten die Erkenntnisse, die im Rahmen einer Masterarbeit (Schabasser 2016) zum Thema gewonnen wurden und das Ergebnis der Triangulierung folgender empirischer Befunde darstellen:

- Der phänomenologischen Erfassung Offener Technologielabore als Dritte Orte durch die Teilnahme an einer 1-tägigen Exkursion im Rahmen einer Lehrveranstaltung der Universität für Bodenkultur Wien zu 2 Otelo-Standorten in Österreich im Juni 2015

- Der Ergebnisse einer leitfadengestützten mündlichen Befragung des Gründers von Otelo im November 2015 zwecks vertiefender Erörterung des konzeptionellen Hintergrundgedankens und der Einordenbarkeit von Otelo in den Stufenbau der Angebote der Daseinsvorsorge sowie der Vorbereitung der Befragung der Otelo-Standortsprecher*innen. Der Fragenkatalog für das Interview umfasste 37 - davon 34 offene - Fragen und gliederte sich in folgende 5 Themenblöcke (vgl. Schabasser 2016, S. 12-13): 1. die Entstehungsgeschichte von Otelo, 2. der Prozess der (räumlichen) Verbreitung und die räumliche Umsetzung von Otelo, 3. die Unterstützung des Innovationsprozesses durch Otelo, 4. die raumstrukturelle Einbindung von Otelo in die Standortgemeinden und 5. das persönliche Fazit zum Erfolg von Otelo

- Der Ergebnisse der mündlichen Befragung der OteloStandortsprecher*innen im Winter 2015/2016, im Rahmen derer die Entstehungsgeschichte der Otelos geschildert und ein Überblick über die momentanen Aktivitäten von Otelo in den jeweiligen Standortgemeinden gegeben wurde

- Des Beobachtungsprotokolls zum Treffen der OteloStandortsprecher*innen im Januar 2016 in Oberöster- 
reich. Dabei konnte ein erster Eindruck über die Art und Weise der ,internen“ Erörterung aktueller Entwicklungen in den Otelo-Standortgemeinden bzw. in den Otelos selbst gewonnen werden

Da weder Jahresberichte noch Vereinsdokumentationen zu den standortspezifischen Aktivitäten, respektive der Einschätzung der Erfolge, von Otelo vorliegen, mussten Primärdaten erhoben werden.

Zur Feststellung etwaiger Veränderungen im Angebotsprofil und der Bedeutung von Otelo in der jeweiligen Standortgemeinde führten die Autor*innen dieses Beitrags im Herbst 2019 eine nachfassende Befragung der OteloStandortsprecher*innen durch.

Es wurden alle Standortsprecher*innen der bis zum damaligen Zeitpunkt in Österreich etablierten Otelo-Standorte -7 in Oberösterreich und 2 in Kärnten - mündlich in den Otelo-Standortgemeinden befragt. In 3 von 9 Fällen wurde das Interview mit 2 Vertreter*innen des Standortsprecher*innenteams geführt.

Der Interviewleitfaden umfasste 40 Fragen - darunter 31 offene Fragen -, leitete sich aus der Dokumentenanalyse relevanter Fachliteratur, des Otelo-Handlungsbuchs sowie aus den Erkenntnissen aus dem Gespräch mit dem OteloGründer $\mathrm{ab}$ und war in folgende 4 Themenblöcke gegliedert (vgl. Schabasser 2016, S. 14):

- die Gründung und Umsetzung von Otelo,

- die raumstrukturelle Einbindung von Otelo in die Standortgemeinde

- der Mehrwert von Otelo für die Standortgemeinde und die im Otelo aktiv engagierten Personen

- die Bedeutung von Otelo für die Unterstützung von (kleinräumigen) Innovationsprozessen.

Der Fragenkatalog für das Interview mit dem Standortsprecher des mittlerweile aufgelösten Otelo-Standorts wurde um die Frage nach den Gründen für das Ende der Aktivitäten erweitert.

Die Befragung der Standortsprecher*innen erfolgte diesmal schriftlich. Der Fragenkatalog umfasste 7 Fragen, darunter 2 geschlossene Fragen. Die Frage nach den Verschiebungen in den inhaltlichen Schwerpunkten des jeweiligen Otelo zwischen 2016 und 2019 bezog sich auf die standortspezifischen Angebotsportfolios im Jahr 2016 und war daher speziell auf die Otelo-Standortgemeinden zugeschnitten. An der zweiten Befragung beteiligten sich trotz des Miteinander-Bekanntseins, der telefonischen Vorbesprechung der zweiten Befragung und der Erinnerung nach Aussendung der Fragebögen nur 4 der 8 Otelo-Standortsprecher*innen, darunter 3 aus Oberösterreich. Diese übermittelten per E-Mail neben den ausgefüllten Fragebögen auch die Einverständniserklärung zur anonymisierten Verwendung ihrer Ausführungen im Sinne der Daten- schutzgrundverordnung der Europäischen Union. Im Falle eines Otelo-Standorts retournierten 2 Vertreter*innen des Standortsprecher*innenteams jeweils einen ausgefüllten Fragenkatalog.

Die übrigen 4 Standortsprecher*innen gaben keine weitere Rückmeldung.

Um den Anforderungen einer multiperspektivischen Einschätzung des Mehrwerts von Otelo als Drittem Ort Rechnung zu tragen, entschieden sich die Autor*innen dieses Beitrags dazu, die Perspektive der Kommunen mittels Befragung jener Bürgermeister*innen mitaufzunehmen, die (bereits) im Jahr 2016 einer Otelo-Standortgemeinde vorstanden. Die Idee dahinter war, bezogen auf das Jahr 2019 vertiefende Einblicke über den Beitrag von Otelo zur Befriedigung von sich verändernden Infrastrukturbedarfen infolge des demografischen Wandels und der Bedeutung von Otelo in der Koproduktion in der Daseinsvorsorge zu erhalten.

Der Fragenkatalog für die Bürgermeister*innen bestand aus 7 offenen Fragen und fokussierte auf folgende Aspekte:

- die Herausforderungen der Otelo-Standortgemeinde im Zusammenhang mit der Gemeindeentwicklung,

- den Beitrag von Otelo zur Gemeindeentwicklung und die die Einordnung von Otelo in den Stufenbau der Angebote der Daseinsvorsorge unter Berücksichtigung ehrenamtlicher Angebote,

- den Status quo zu Otelo in der Gemeinde und die Unterstützung von Otelo durch die Gemeinde,

- die Reflexion der anfänglichen Erwartungen der Bürgermeister*innen an Otelo, der Erfüllung der Erwartungen und weiterführende Anmerkungen zur Bedeutung von Otelo für die Gemeindeentwicklung.

Im Januar 2019 erfolgte die erste telefonische Kontaktaufnahme, im Rahmen derer um Unterstützung dieser Eigenforschung ersucht und die Befragungsform gemeinsam festgelegt wurde. Im März 2019 wurde der Fragenkatalog samt Begleitschreiben und Informationsblatt über den anonymisierten Umgang mit personenbezogenen Informationen im Sinne der Datenschutzgrundversorgung der Europäischen Union per E-Mail an die Bürgermeister*innen übermittelt. In der telefonischen Vorbesprechung erklärten sich die Bürgermeister*innen dazu bereit, für weiterführende Fragen bzw. die Erörterung etwaiger Unklarheiten in den schriftlichen Ausführungen zur Verfügung zu stehen.

Fünf der 9 Bürgermeister*innen retournierten den ausgefüllten Fragenkatalog sowie die Einverständniserklärung im April 2019 per E-Mail. Die Erinnerung im November 2019 bzw. Januar 2020 erbrachte die Beteiligung weiterer 3 Bürgermeister.

Die Gespräche wurden mit dem Einverständnis der Befragten aufgezeichnet, sinngemäß transkribiert, zentrale Aussagen wortwörtlich übernommen und ebenso wie die 
schriftlichen Ausführungen nach der Methode von Gläser und Laudel (2009) inhaltsanalytisch ausgewertet.

Die Einschätzung des Mehrwerts von Otelo für die kommunale Entwicklung und die Erfüllung der Merkmale als sogenannte Dritte Orte nach Oldenburg (1999) erfolgte

- einerseits unter Zugrundelegung verschiedener Definitionen des Begriffs „Innovation“ nach Rogers (2003), soziale Innovation nach Howaldt und Schwarz (2010) und Kreativwirtschaft nach Bachinger et al. (2017) sowie unter Berücksichtigung der Reichweite von Innovation nach Heiler (2008),

- andererseits durch den Vergleich mit den im Otelo-Handlungsbuch definierten Wirkungszielen und Anforderungen an Otelo (Hollinetz et al. 2012) sowie des 2015 publizierten Otelo-Wirkungsberichts (Waser et al. 2015). Die inhaltsanalytische Auswertung erfolgte in Anlehnung an die Methode von Boyatzis (1998).

Rogers (2003, S. 11) versteht unter Innovation Folgendes ,An innovation is an idea, practice or object that is perceived as new by an individual or other unit of adoption. It matters little, so far as human behaviour is concerned, whether or not an idea is ,objectively " new ... If the idea seems new to the individual, it is an innovation." Howaldt und Schwarz definieren soziale Innovationen als ,,... von bestimmten Akteuren bzw. Akteurskonstellationen ausgehende intentionale, zielgerichtete Neukonfiguration sozialer Praktiken in bestimmten Handlungsfeldern bzw. sozialen Kontexten, mit dem Ziel, Probleme oder Bedürfnisse besser zu lösen bzw. zu befriedigen, als dies auf der Grundlage etablierter Praktiken möglich ist" (Howaldt und Schwarz 2010, S. 89). Heiler wiederum bezieht neben dem Neuigkeitsaspekt auch die Reichweite der Innovation in die Definition mit ein und unterscheidet folglich zwischen inkrementeller Innovation und profunder Innovation. Erstere umfasst Verbesserungen und adaptive Neuerungen von Produkten, Dienstleistungen, Verfahren oder Organisationsformen und steigert unter anderem die Kosteneffizienz. Zweitere hingegen bezieht sich auf neue Produkte, Dienstleistungen, Prozesse und Organisationsformen, die eine grundlegende Veränderung von Märkten und sozialen Systemen bewirken (Heiler 2008, S. 92-93). Schließlich wird zur Beurteilung des Beitrags von Otelo zur Kreativwirtschaft folgende Definition nach Bachinger et al. (2017, S. 11) verwendet: Kreativwirtschaft umfasst „erwerbsorientierte Unternehmen, die sich mit der Schaffung, Produktion, und (medialen) Distribution von kreativen und kulturellen Gütern sowie Dienstleistungen beschäftigen." Dazu zählen Unternehmen in den Bereichen Architektur, Buch- und Verlagswesen, Design, Filmwirtschaft, darstellende Kunst, Musikwirtschaft, Radio und TV, Software und Games, Werbung sowie Bibliotheken, Museen, botanische und zoologische Gärten.

\section{Das Offene Technologielabor als Dritter Ort}

Der Vereinsname „Offenes Technologielabor“ beruht auf den folgenden Grundgedanken: Otelo ist „offen“ hinsichtlich Zeit, Ziel und Thema des Engagements und hinsichtlich der Zielgruppen; „Labor“ unterstreicht die Wichtigkeit des Ausprobierens und Experimentierens und „Technologie“ ist im Sinne der ursprünglichen, weit gefassten griechischen Bedeutung der Kunstfertigkeit etwas Bestimmtes $z u$ erreichen, zu verstehen. Vergleicht man diese und weitere zentrale Komponenten des Otelo-Konzepts mit den 8 beschriebenen Kerncharakteristika Dritter Orte nach Oldenburg (1999), wird die Überschneidung der beiden Konzepte schnell ersichtlich:

1. Otelo als neutraler, offener Ort: „Die Offenen Technologielabore leben von der Idee Menschen einen offenen Raum für kreative und technische Aktivitäten zu ermöglichen. ... [Otelo] versteht sich als eigenes, lebendiges System, und läßt sich von bestehenden Gesellschaftssystemen und Institutionen nicht vereinnahmen, damit es Freiheiten hat unser Gesellschaftssystem in Richtung Nachhaltigkeit und Menschenwürde zu beeinflussen“ (Hollinetz et al. 2012, S 7).

2. Otelo als Begegnungsort auf Augenhöhe: In Otelo Workshops ,steht das gemeinsame Schaffen auf Augenhöhe im Vordergrund" (Hollinetz et al. 2012, S. 78).

3. Otelo als Ort des Dialogs und der Beziehung: „Damit sich Ideen und Wissen durch ,Sharing 'vervielfältigen können, braucht es Bindungen und Beziehungen zwischen Menschen, die wie ein Schmiermittel wirken. Es braucht Kommunikation und Interaktion, sowohl innerhalb der eigenen ,kleinen Welt‘ (Familie, Freundeskreis, Verein ...) als auch zu Menschen außerhalb dieses engeren Kreises. Es braucht ,Sozialkapital ““ (Hollinetz et al. 2012, S. 70).

4. Otelo als erreichbarer und zugänglicher Ort: Die Räumlichkeiten der Offenen Technologielabore sollten laut Konzept leicht zugänglich sein. Wenn ein Raum nur von einer Gruppe verwendet wird, sollte es auch möglich sein, ihn abzusperren. $\mathrm{Zu}$ diesem Zweck werden z. B. Schlüsselboxen verwendet. (Hollinetz et al. 2012, S. 68-69).

5. Die Otelo Magic 5 als Stammklientel: „,... eine (kleine) Gruppe von Menschen (vgl. Magic 5) willigt ein, ein erstes Stück des Entwicklungsweges mitzugehen ... Diese Gruppe hat auch Lust am Aufbau und am Erhalt des Organisationsrahmens mitzuarbeiten. Durch den Ansatz von [Otelo], frei von externen Systemen zu bleiben, setzt die Basisorganisation ein ehrenamtliches Engagement voraus" (Hollinetz et al. 2012, S. 54).

6. Das Otelo-Raumkonzept zur Förderung von Interaktion und Dialog: ,Nach den bisherigen Erfahrungen kann ein 
OTELO Standort gut ,gedeihen“ wenn mind. Ca $250 \mathrm{~m}^{2}$ Fläche am Beginn zur Verfügung stehen. Basis eines Standortes sollte der Aufenthaltsraum mit Küche, die Gemeinschaftswerkstätte, ein Raum für Workshops/Jam Sessions und mindesten 3 Räume, wo Nodes entstehen können." (Hollinetz et al. 2012, S. 69).

7. Otelo als Spiel- und Experimentierort: „,[Otelos] bilden Gemeinschaften für experimentierfreudige Akteure/ innen und für gesellschaftspolitische Initiativen. Sie laden zur aktiven Beteiligung ein. [Otelo] bietet einen Organisationsrahmen, der die Beteiligten bei der Entwicklung von Ideen und bei experimentellen Aktivitäten unterstützt" (Hollinetz et al. 2012, S. 7).

8. Otelo als Wohlfühlort: „Otelo handelt im Bewußtsein wertschätzender Anerkennung persönlicher Potenziale und seiner freien Entfaltung" (Hollinetz et al. 2012, S. 8). Dabei bietet Otelo einen informellen Rahmen, in dem Wert auf die Gemütlichkeit und das „,Wohlfühlen“ gelegt wird: In Offenen Technologielaboren ,,muss“ nichts passieren - es ,kann“ etwas passieren (Schabasser 2016, S. 85).

Grundlegende Richtlinien für die Gründung eines Otelo-Standorts sind im Otelo-Handlungsbuch (Hollinetz et al. 2012) beschrieben. Dabei besteht die materielle Basis zur
Gründung eines Standorts aus kostenlos vom Gemeinderat zur Verfügung gestellten Räumlichkeiten (siehe auch Punkt 6) sowie Internet.

\section{Raumplanerische Herausforderungen in den untersuchten Gemeinden}

Die ersten beiden Standorte des Offenen Technologielabors entstanden 2010 in Gmunden und Vöcklabruck, 2012 und 2014 folgten 5 weitere Standorte in Oberösterreich. Im Jahr 2015 breitete sich der Verein mit 2 weiteren Standorten in den Süden Österreichs nach Kärnten aus. Abb. 1 stellt diese Standorte (inklusive des wieder aufgelassenen Otelos in Scharnstein) auf der Österreichkarte „Urban-Rural-Typologie“ der Statistik Austria (2016) dar und gibt so einen Einblick in ihren räumlichen Kontext. Bei den 8 untersuchten Otelo-Standorten handelt es sich um 5 urbane bzw. regionale Zentren und um 4 Gemeinden im ländlichen Raum im Umland von Zentren.

Zusätzliche Informationen zum räumlichen Kontext der untersuchten Otelo-Standorte liefern die Perspektiven der Bürgermeister*innen auf die Herausforderungen ihrer jeweiligen Standortgemeinden. Diese sind im Folgenden in 4 Kategorien zusammengefasst:

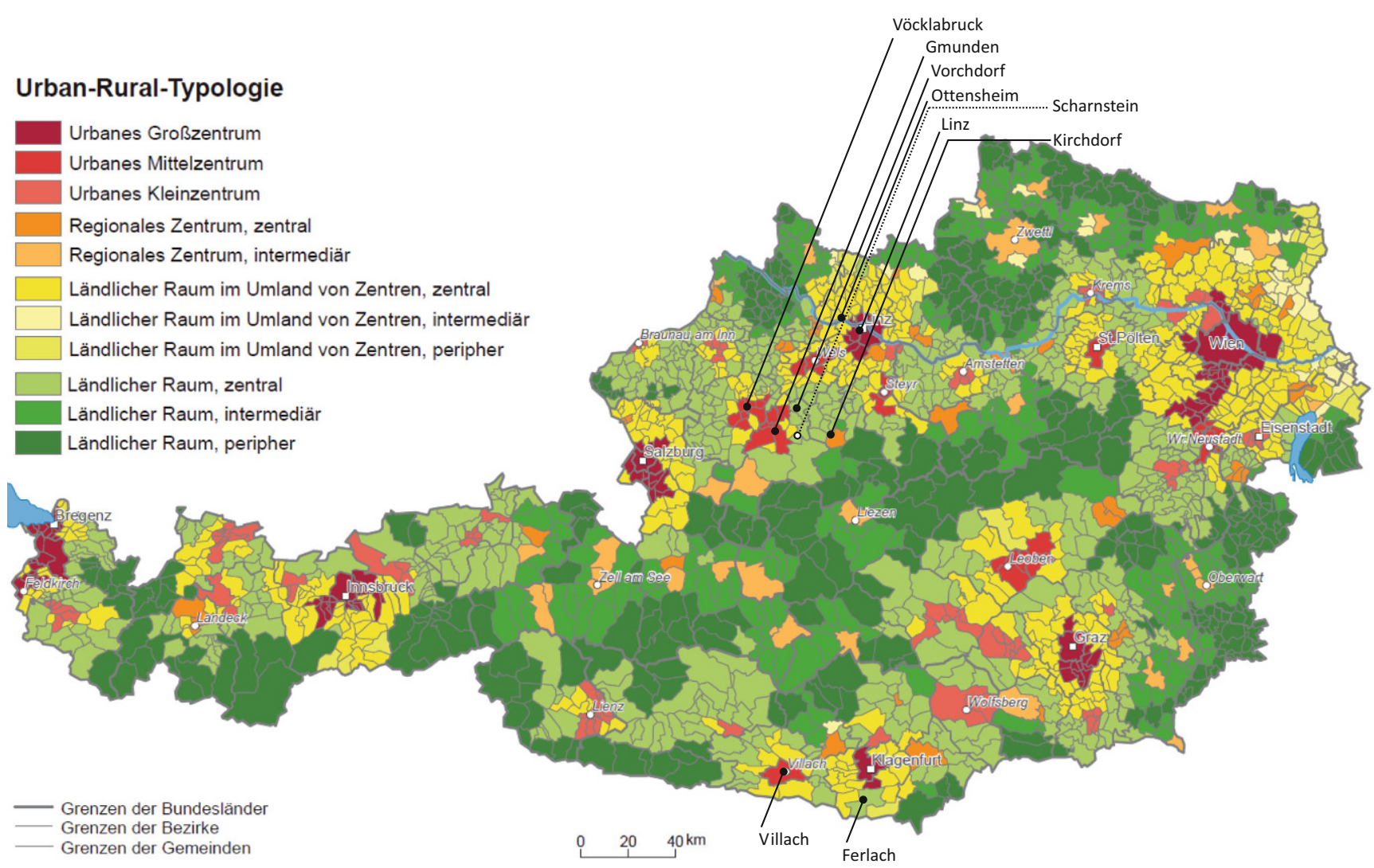

Abb. 1 Urban-Rural-Typologie der Statistik Austria inklusive Standorte der ersten 9 Otelos. (Quelle: verändert nach Statistik Austria 2016). Anmerkung: Der Otelo-Standort in Scharnstein wurde inzwischen aufgelöst 
1. Demografische Herausforderungen: Alterung der Bevölkerung, Abwanderung der Jugend, geringes Bevölkerungswachstum an einem Standort/schnelles Bevölkerungswachstum an einem anderen Standort, hohe Fertilität (,,anhaltender Babyboom“ [Bürgermeister*in \#4, 2020]) sowie damit einhergehende Herausforderungen (Bereitstellung eines adäquaten Kinderbetreuungsangebots sowie mobiler und stationärer Betreuung für ältere Menschen)

2. Herausforderungen in der örtlichen Raumplanung: Hochwasserschutz, kaum Grundstücksreserven, Verkehrsmanagement (Pendlermanagement, Förderung umweltfreundlicher Verkehrsmittel und Ausbau des öffentlichen Verkehrs)

3. Herausforderungen im Zusammenhang mit der Standortqualität: Abwanderung von Fachkräften, Übergang von Industriestadt zur ,Innovationsstadt“, Unterstützung lokal angesiedelter internationaler Unternehmen, Abwanderung von Geschäften aus der Innenstadt sowie in einem Fall die Nähe der Gemeinde zu einem städtischen Zentralraum

4. Institutionelle und sozioökonomische Herausforderungen: angespannter Kommunalhaushalt, Umsetzung von Klimaschutzmaßnahmen, mangelndes Übereinkommen in Vereinen, Entsolidarisierung der Gesellschaft, Ausbau eines regionalen und nachhaltigen Lebensmittelangebots

Wie der erste Otelo-Wirkungsbericht (Waser et al. 2015, S. 9) zeigt, sind es gerade die Herausforderungen der demografischen Entwicklung und der Standortqualität, für welche Otelo postuliert, einen Lösungsansatz zu bieten: nämlich die Herausforderungen einer alternden Bevölkerung,
Braindrain, neue Stadt-Land-Beziehungen sowie ein Systemwandel in der Wirtschaft.

\section{Die Offenen Technologielabore in der Praxis: Aktive Gruppen 2016}

Um eine Einschätzung darüber treffen zu können, inwieweit die Offenen Technologielabore die oben skizzierten Herausforderungen kommunaler Entwicklung adressieren und die Gemeinden in der Bereitstellung von Dienstleistungen und Infrastruktur ergänzen, werden die im Rahmen von Otelo gegründeten Gruppen (nachfolgend auch als „Nodes“ für „,Knotenpunkte“ im Otelo-Netzwerk bezeichnet) im Folgenden den Daseinsgrundfunktionen der Münchner Schule für Sozialgeografie (Werlen 2000, S. 175-177) zugeordnet (siehe Abb. 2): Arbeit, Bildung, Erholung, Gemeinschaftsleben, Teilnahme am Verkehr, Ver- und Entsorgung sowie Wohnen.

Im Januar 2016 betrug die Gesamtanzahl der Otelo-Nodes an den 9 untersuchten Standorten 52. Abb. 2 zeigt, dass 22 der 52 Gruppen den Zweck der Erholung hatten. Zwölf dieser Gruppen beschäftigten sich mit kreativen Fähigkeiten (wie kreatives Schreiben und Rappen), Kunsthandwerk sowie IT, Elektronik oder nachhaltigem Lebensmittelkonsum (Genuss-Node). Diese Gruppen werden aufgrund ihres Hobby-Charakters vorrangig der Kategorie Erholung zugeordnet, und aufgrund vorhandener Bildungsaspekte überlappend mit dem Bereich Bildung dargestellt. Andere Gruppen in der Kategorie Erholung betonen Gemeinschaftlichkeit, indem sie Aktivitäten anbieten, die auch individuell oder privat ausgeübt werden könnten, wie beispielsweise Gartenarbeit, Musizieren, Malen (Abb. 3), Brettspiele oder
Abb. 2 Otelo-Gruppen im Januar 2016. ( $n=52,9$ OteloStandorte; Quelle: eigene Darstellung)

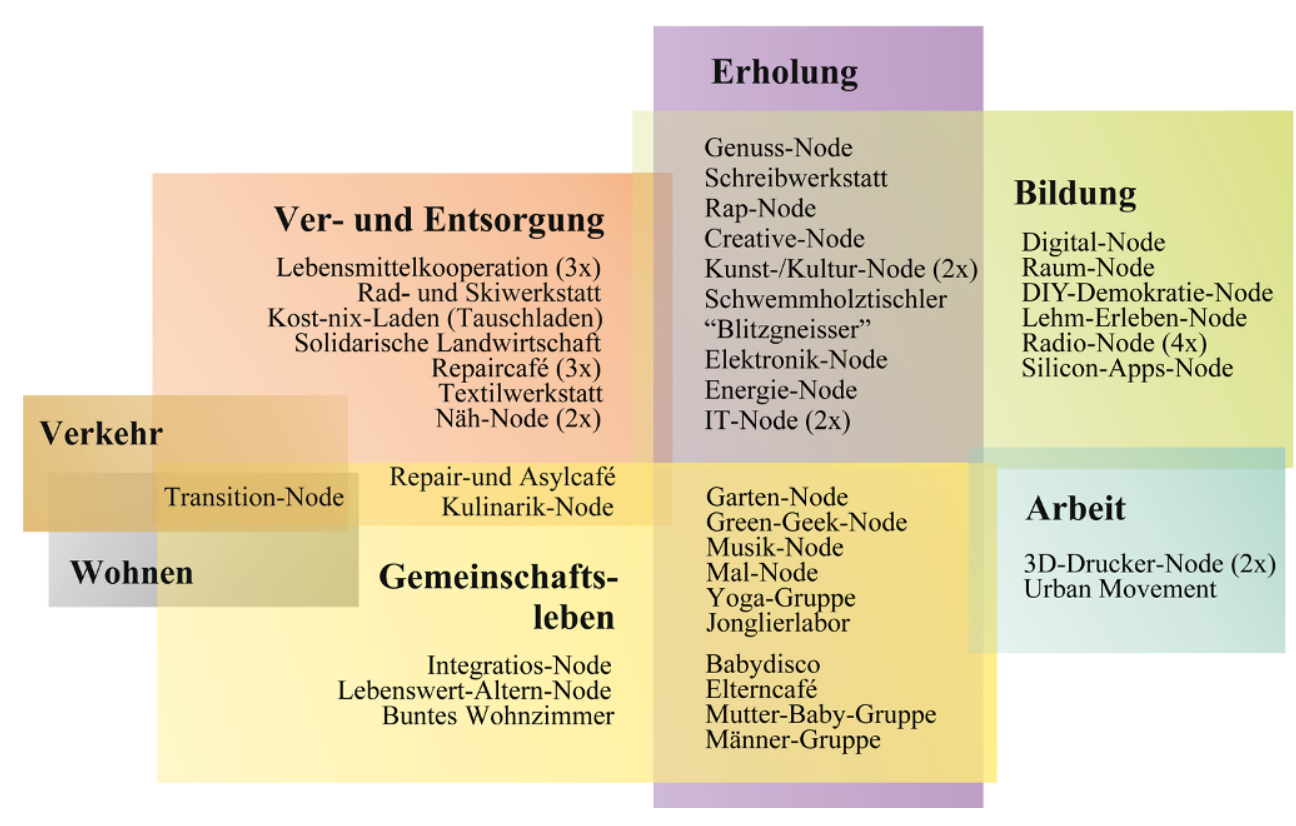




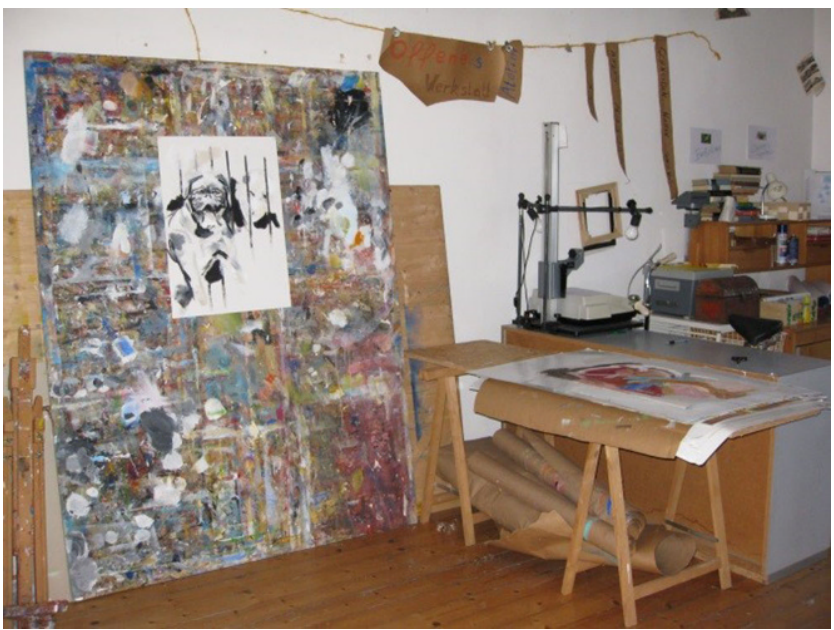

Abb. 3 Malwerkstatt im Otelo Ottensheim. (Quelle: Aufnahme der Autor*innen)

Sport. Schließlich gab es 4 Gruppen, die auf gemeinsamen Bedürfnissen je nach Lebenssituation basieren (z. B. das Elterncafé, die Mutter-Kind-Gruppe oder die Männergruppe).

Die Kategorie Gemeinschaftsleben umfasst 3 Gruppen, darunter eine, die sich mit den Bedürfnissen älterer Menschen befasst; eine Gruppe, die den Austausch mit Personen im Asylantragsverfahren fördert, und schließlich das sogenannte „Bunte Wohnzimmer“, welches zu einem unverbindlichen, geselligen Beisammensein einlädt.

Im Bereich Ver- und Entsorgung brachte Otelo bis 2016 3 Lebensmittelkooperationen hervor, welche den Konsum lokaler landwirtschaftlicher Produkte unterstützen und organisieren sowie eine solidarische Landwirtschaft ("Gemüse-SoLaWi,,) - ein gemeinschaftlich bewirtschaftetes Stück Land zur Produktion von Gemüse. Außerdem sind dem Bereich der Ver- und Entsorgung folgende Gruppen zuzuordnen: 3 Nähinitiativen, die die Heimproduktion von Textilar-

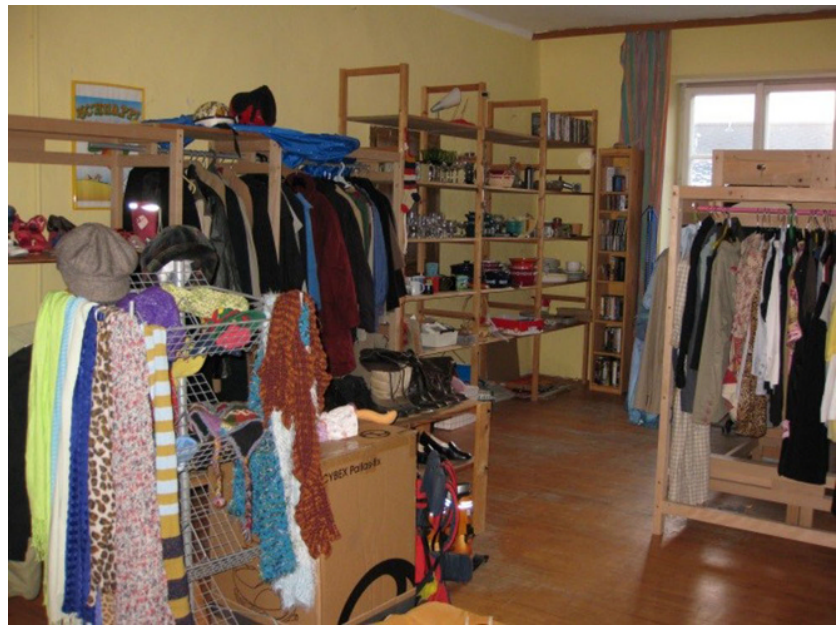

Abb. 4 Kost-nix-Laden im Otelo Ottensheim. (Quelle: Aufnahme der Autor*innen) tikeln fördern; eine Tauschbörse („Kost-nix-Laden“, siehe auch Abb. 4); eine Fahrrad- und Skiwerkstatt, welche die Reparatur und Wartung von Fahrrädern und Skiern erleichtert; sowie mehrere Reparaturcafés, in denen kaputte Gegenstände mit gegenseitiger Unterstützung repariert werden. Ein weiteres Reparaturcafé wird Reparatur- und Asylcafé genannt, weil es den Austausch mit Menschen unterstützt, die sich im Asylantragsverfahren befinden und über Fachwissen im Bereich der Elektronik verfügen, wodurch die Gruppe sowohl sozialen Zusammenhalt als auch Umweltschutz durch Abfallvermeidung zu fördern versucht. Auch der Kulinarik-Node lässt sich beiden Bereichen zuordnen, da er mit der Aufbereitung von Lebensmitteln, die sonst weggeworfen würden, in Kombination mit Lesungen oder Vorträgen gleichzeitig Abfallvermeidung und sozialen Austausch unterstützt. Der Transition-Node beschäftigt sich mit einer Vielzahl von Themen wie Gemeinschaftsgärten, solidarischer Landwirtschaft, gemeinsames Wohnen, gemeinsames Altern, Carsharing und nachhaltige Mobilität und deckt somit die Bereiche Verkehr, Wohnen und Gemeinschaftsleben ab.

Unter der Daseinsgrundfunktion Bildung sind ein Abiturprojekt zur Erstellung einer Onlinezeitung (Digital-Node), 1 Gruppe zur Programmierung einer virtuellen Kunstausstellung (Raum-Node), 1 Gruppe zur Beschäftigung mit Diskussions- und Entscheidungsformaten (DIY-Demokratie-Node), 1 Gruppe zur Beschäftigung mit dem Werk- und Baustoff Lehm (Lehm-Erleben-Node), 4 Freie Radios (siehe auch Abb. 5) sowie der Silicon-Apps-Node (mit Programmierkursen für Kinder und Computerkursen für Senior*innen) zu finden.

Zur Daseinsgrundfunktion Arbeit werden 2 3D-Druckergruppen zugeordnet, da aus ihnen 2 Unternehmen zur Produktion von 3D-Druckern entstanden (siehe auch Abb. 6).

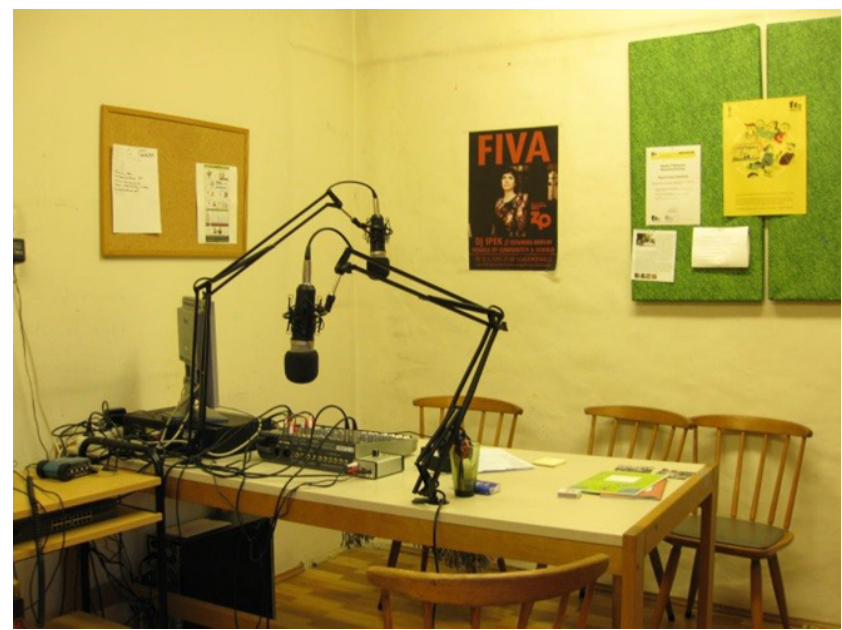

Abb. 5 Radiostudio im Otelo Ottensheim. (Quelle: Aufnahme der Autor*innen) 


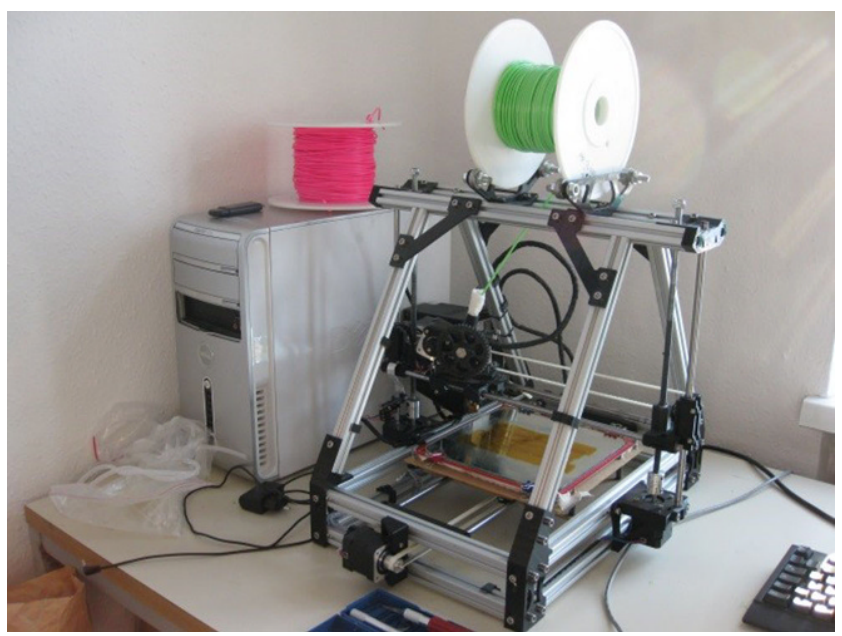

Abb.6 3D-Drucker im Otelo Vöcklabruck. (Quelle: Aufnahme der Autor*innen)

Auch die Tanzgruppe Urban Movement wurde zum Zeitpunkt der Interviews für Auftritte gebucht.

\section{Die Offenen Technologielabore 2019 - Beständigkeit der Aktivitäten, identifizierte Mehrwerte und (un-)erfüllte Erwartungen}

Von den im Januar 2016 befragten Standortsprecher*innen der 9 ersten Otelo-Standorte antworteten Standortsprecher*innen an 4 Otelo-Standorten auf eine Folgeumfrage im September 2019. Abb. 7 veranschaulicht die Veränderung der Gruppen an diesen 4 Standorten im Vergleich zu 2016: Von den 25 ursprünglich erfassten Gruppen an den 4 Standorten wurden 5 Gruppen aufgelöst (in Abb. 7 durchgestrichen) und 12 neue Gruppen gegründet (siehe fett markierte Gruppen), was 32 bestehende Gruppen ergibt.
Diese Bestandsaufnahme veranschaulicht, dass die Mehrheit der Otelo-Gruppen zwischen 2016 und 2019 aktiv blieb und dass die Gesamtheit der Otelo-Aktivitäten tendenziell zunahm. Wurden Gruppen beendet, dann aus folgenden Gründen: Die Aktivitäten wurden nur von einer Person organisiert, welche aufgrund von privaten oder beruflichen Veränderungen keine Zeit mehr für die OteloGruppe hatte, oder die Person verlor das Interesse daran, die Gruppe weiterzuführen. Ein weiterer Grund war eine zu geringe Nachfrage nach bestimmten Aktivitäten.

Das Engagement der Otelo-Beteiligten entwickelte sich zwischen Anfang 2016 und Ende 2019 laut Standortsprecher*innen unterschiedlich: von alternierend über stabil bis hin zu positiv, wie in folgenden Zitaten aus der Befragung sichtbar wird:

,... einige wichtige Personen haben sich zurückgezogen, andere aber haben sich verstärkt engagiert, neue Interessierte sind hinzugekommen" (Standortsprecher*in \#2, 2019).

„[Das Engagement] ist gleichbleibend hoch. Viele sind schon lange dabei und deren Engagement verhält sich wellenförmig, gleicht sich aber gegenseitig aus." (Standortsprecher*in \#4, 2019).

Insgesamt deuten diese Ergebnisse darauf hin, dass sich die Offenen Technologielabore zwischen Januar 2016 und September 2019 positiv entwickelt haben, da an den verschiedenen Otelo-Standorten neue Gruppen und Initiativen gegründet wurden. Im Zeitverlauf hat sich gezeigt, dass sich rückläufiges Engagement bestimmter Mitglieder mit vermehrtem Engagement anderer Otelo-Mitglieder ausgleicht bzw. an manchen Standorten insgesamt eine positive Tendenz aufweist.

Laut Standortsprecher*innen setzt sich der Mehrwert der im Herbst 2019 bestehenden Otelo-Gruppen und Aktivitäten hauptsächlich aus der Förderung eines ressourcenscho-
Abb. 7 Otelo-Gruppen im September 2019. ( $n=25$, 4 OteloStandorte; Quelle: eigene Darstellung)

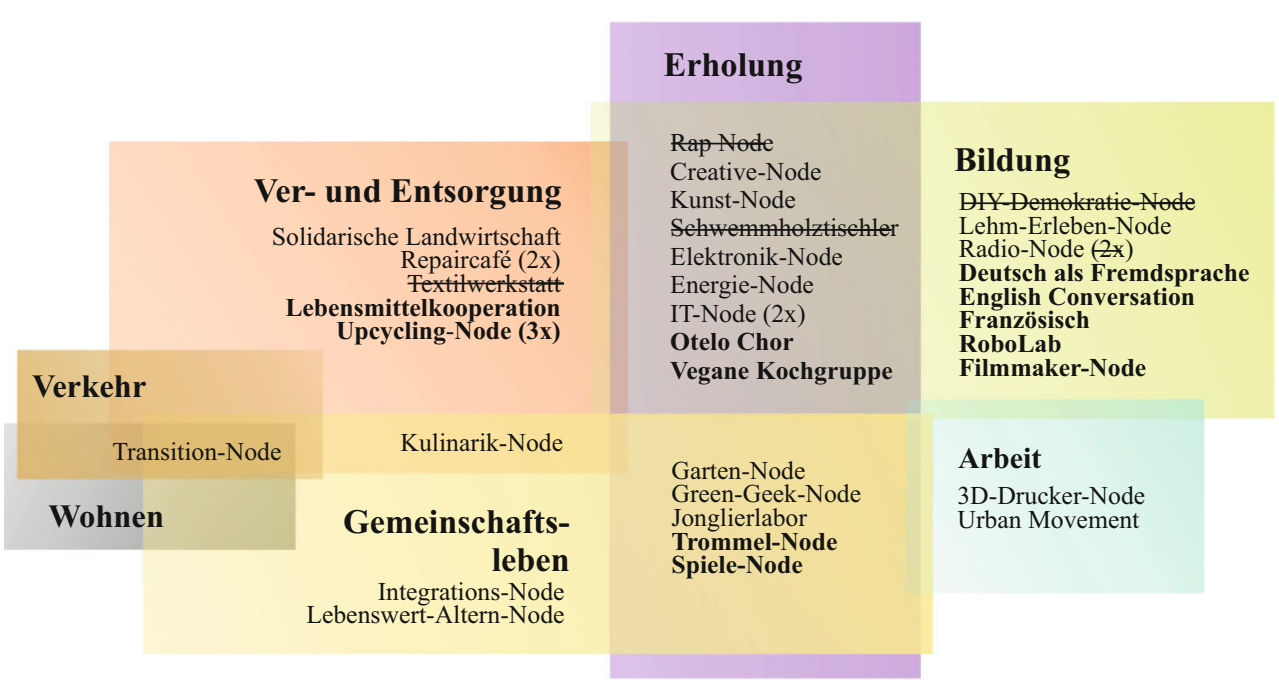




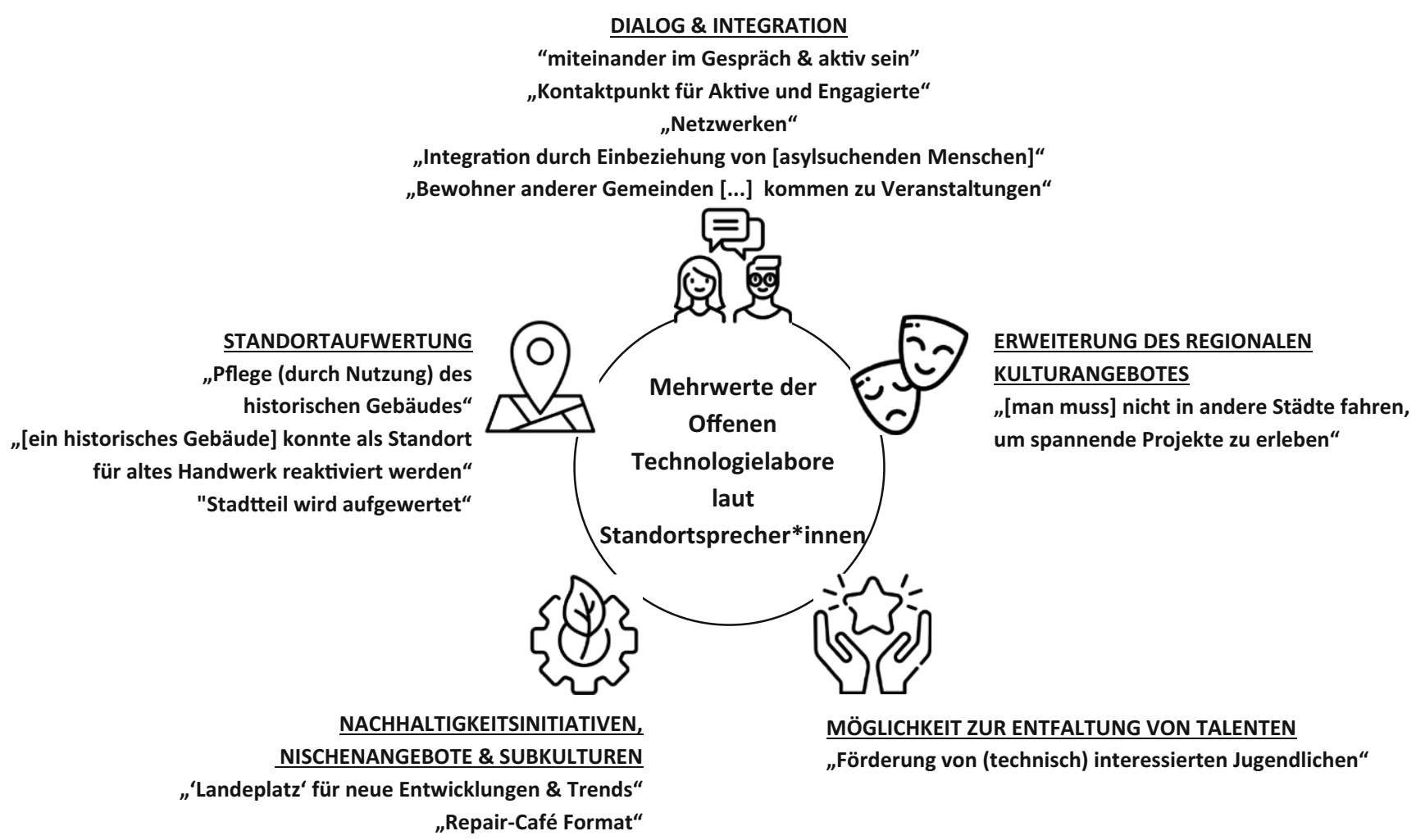

Abb. 8 Mehrwert der Offenen Technologielabore laut Standortsprecher*innen. (Quelle: eigene Darstellung; Quelle der verwendeten Symbole: Freepik und Good Ware auf www.flaticon.com)

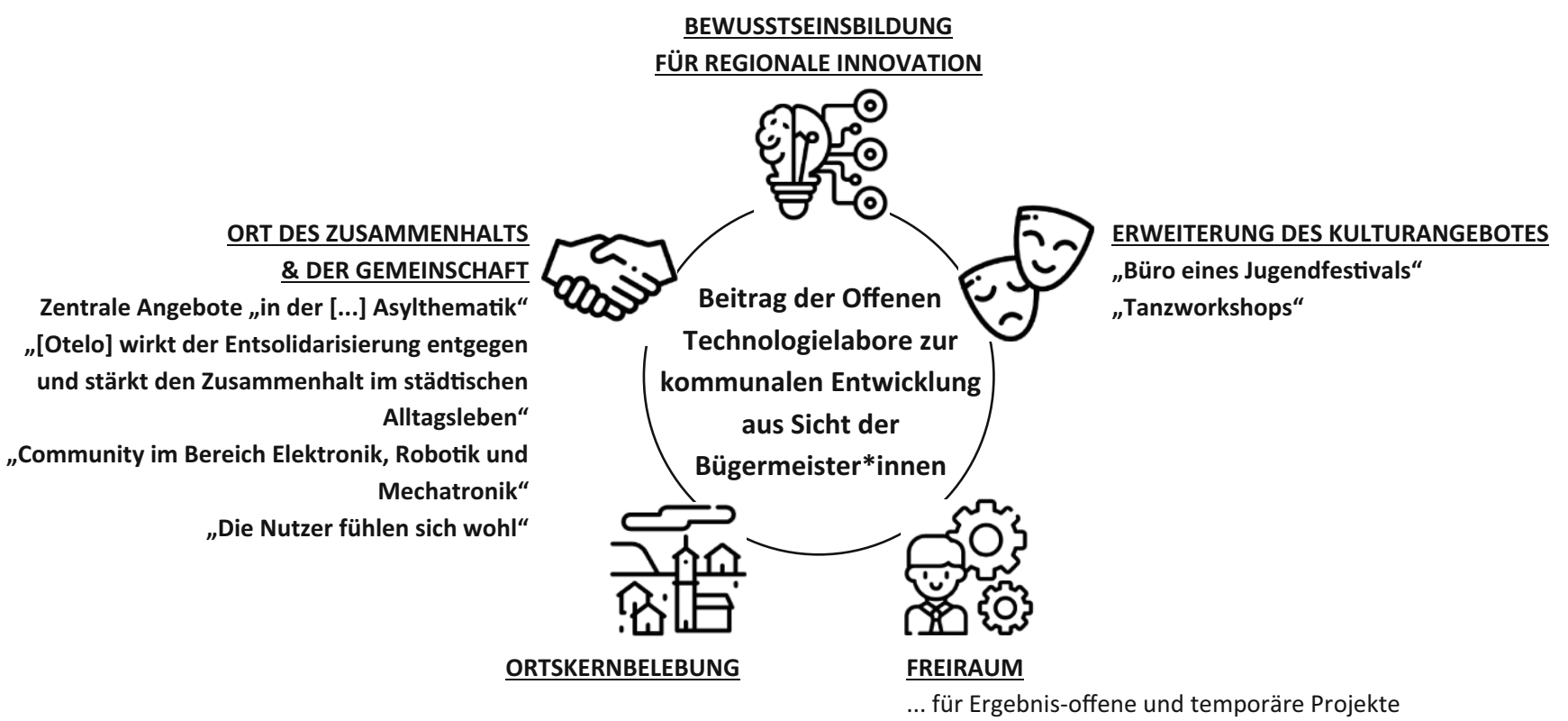

Abb. 9 Beitrag der Offenen Technologielabore zur kommunalen Entwicklung aus Sicht der Bürgermeister*innen. (Quelle: eigene Darstellung; Quelle der verwendeten Symbole: Freepik und Eucalyp auf www.flaticon.com) 
nenden Lebensstils $(n=5)$ sowie neuen Organisationsformen \& neuen sozialen Beziehungen $(n=4)$ zusammen. Darüber hinaus unterstrichen auch 3 Standortsprecher*innen den Beitrag Otelos zur individuellen Lebensqualität \& zur sozialen Inklusion. Zwei Standortsprecher*innen stimmten zu, dass Otelo zur Förderung neuer Produkte \& Serviceleistungen beitrage. Unter ,Sonstiges“ wurde an einem Standort eine imagefördernde Wirkung einer Otelo-Gruppe genannt, wobei nicht spezifiziert wurde, auf wen oder worauf sich diese imagefördernde Wirkung bezieht.

Die Standortsprecher*innen nannten folgende Aspekte, die sie als wertvoll für ihre Gemeinden erachten (siehe Abb. 8.):

Die Bürgermeister*innen betrachten den Beitrag von Otelo zur kommunalen Entwicklung allgemein als positiv. Ihre Antworten reichen von allgemeinen Anliegen bis hin zu sehr spezifischen Beiträgen (siehe Abb. 9):

Die Bürgermeister*innen sehen diese Beiträge weitgehend als komplementär und nicht als überschneidend mit den bereits bestehenden ehrenamtlichen Aktivitäten in ihren Gemeinden. In einem Fall wurde aber die enge Vernetzung von Otelo mit bereits bestehenden sozialen Innovationsprojekten betont.

Was die anfänglichen Erwartungen der Bürgermeister*innen der 9 ursprünglich befragten Otelo-Standorte anbelangt, so erhofften sich diese von Otelo vor allem einen Raum zum Netzwerken, Aktivitäten für unterschiedliche Altersgruppen von Jung bis Alt, ein Freizeitangebot für Kinder und Jugendliche sowie einen Raum für Kreativität und soziales Engagement. An einem Standort erfüllte sich die Erwartung eines neuen Zugangspunkts zur Bevölkerung. In einem weiteren Fall wurde der erhoffte Effekt hinsichtlich eines Bildungsauftrags im Bereich Technik und Naturwissenschaften bestätigt. Ein weiterer Bürgermeister erklärte, er hatte neben anderen Schwerpunkten ebenso einen Technikfokus erwartet, welcher sich jedoch nicht einstellte.

\section{Diskussion}

Wie das Konzept der Dritten Orte forciert auch Otelo Begegnungen von Menschen mit ähnlichen Interessen, die sich sonst nicht getroffen hätten, um das gesellschaftliche Gefüge an den jeweiligen Standorten zu stärken und so die Initiierung formellerer Kooperationen zu fördern. Zudem sind Offene Technologielabore Orte des Austauschs von Neuigkeiten im Sinne Dritter Orte, da sie - wie von einer bzw. einem der befragten Standortsprecher*innen formuliert als Sammelorganisation für ,Nischenangebote und Subkulturen“ beziehungsweise als „Landeplatz für neue Entwicklungen und Trends" (Standortsprecher*in \#3, 2019) fungieren. Hinsichtlich ihrer räumlichen Dimension (urban - sub- urban - ländlich), der (sozialen) Innovationsträchtigkeit der Offenen Technologielabore, ihres Beitrags zur Wirtschaft bzw. Kreativwirtschaft sowie ihrer Beständigkeit ist folgendes Fazit zu ziehen:

\section{Räumliche Dimension von Otelo}

Was seine räumliche Dimension betrifft, so erhebt das Konzept der Offenen Technologielabore den Anspruch, in ländlichen Räumen zu wirken. In der Umsetzung hat sich gezeigt, dass es sich bei den Otelo-Standorten um urbane bzw. regionale Zentren und Gemeinden im Umland von Zentren handelt. Dieses Ergebnis der Ersterhebung aus 2016 bestätigen auch die derzeitigen Standorte laut Otelo Website: Von den 24 neuen Standorten in Österreich sind 16 Gemeinden urbane bzw. regionale Zentren, weitere 4 Gemeinden befinden sich im ländlichen Raum im Umland von Zentren und schließlich gibt es 4 ländliche Standorte außerhalb von Zentralräumen. Diese Ergebnisse lassen vermuten, dass eine kritische Masse an potenziellen Nachfrager*innen zur Gründung eines Otelos relevant ist und dass die Erreichbarkeit des Standorts sowie der Lebensstil potenzieller Otelo-Mitglieder (Pendeln als Bestandteil des Alltags) zu den Erfolgs- bzw. Hemmfaktoren für die Gründung von Offenen Technologielaboren zählen. Die dargestellten raumplanerischen Herausforderungen wurden bisher punktuell von Otelo-Gruppen aufgegriffen. Beispiele aus dem Strauß an themenspezifischen Gruppen sind die Lebenswert-AlternGruppe in Ferlach (Kärnten) oder die Lebensmittelkooperation in Vöcklabruck. Diese Angebote jedoch sind nicht das Ergebnis einer strategischen Abstimmung von standortbezogenen, respektive kommunenspezifischen Defiziten, sondern spiegeln die persönlichen Anliegen und Interessen der Otelo-Mitglieder wider. Daraus lässt sich schließen, dass infrastrukturelle Defizite bzw. Versorgungslücken nicht automatisch von Otelo aufgegriffen bzw. gefüllt werden (können).

\section{Innovation durch Otelo}

Zieht man die eingangs gegebenen Definitionen von Innovation nach Rogers (2003) und Heiler (2008) bzw. von sozialer Innovation nach Howaldt und Schwarz (2010) heran, so sind aus den Offenen Technologielaboren einige inkrementelle Innovationen hervorgegangen. Objektiv neu sind weder Lebensmittelkooperationen noch die Solidarische Landwirtschaft, der Kost-nix-Laden, das Radund Skiamt oder die Repaircafés. Doch handelt es sich im Falle der Lebensmittelkooperationen und der Solidarischen Landwirtschaft um Praktiken, die es in der jeweiligen Gemeinde zuvor nicht gab und in diesem Sinne um soziale Innovationen im Kontext dieser Gemeinden. Im Falle des Kost-nix-Ladens, des Rad- und Skiamts und der Repair- 
cafés ist der innovative Aspekt darin zu sehen, dass die weitverbreiteten Praktiken der Reparatur und Instandhaltung im Rahmen des Vereins erleichtert und unterstützt werden, indem das nötige Werkzeug sowie ein zentraler Sammel- und Abholpunkt für wiederzuverwendende Gegenstände bereitgestellt werden. Die Repaircafés können insofern als innovativ betrachtet werden, da hier das nötige Wissen über die Reparatur von Gegenständen geteilt wird, welches ansonsten größtenteils von kommerziellen Anbieter*innen in Wert gesetzt wird. Auffallend ist, dass all diese inkrementellen sozialen Innovationen versuchen, einem auf Wachstum ausgerichteten Wirtschaftssystem Alternativen entgegenzusetzen.

\section{Beitrag zur (Kreativ-)Wirtschaft von Otelo}

In einem Zeitraum von 9 Jahren entwickelten sich 2 Startup-Unternehmen aus Otelo-Initiativen. Daher bleibt ein substanzieller Beitrag zur Kreativwirtschaft und zum Arbeitsmarkt noch abzuwarten. Wie Abb. 2 veranschaulicht, sind aus Otelo bisher eine Reihe an Gruppen zur kreativen Beschäftigung (wie Nähen, Schreiben, Rappen, Tanzen, Musizieren, Radio-Produktion etc.) hervorgegangen, jedoch ist die Ausübung kreativer Hobbies nicht der Kreativwirtschaft - welche zur Stärkung der lokalen oder regionalen Entwicklung beitragen könnte - gleichzusetzen. Anhand der vorliegenden Ergebnisse ist daher kein Beitrag Otelos zur Lösung der von den Bürgermeister*innen genannten Herausforderungen der Abwanderung von Fachkräften und der Unterstützung lokal angesiedelter internationaler Unternehmen zu erkennen.

\section{Beständigkeit von Otelo}

Durch die Erhebung zu 2 Zeitpunkten ist zu erkennen, dass Otelo-Gruppen über mehrere Jahre hinweg aktiv bleiben können. Das Engagement einzelner Personen kann sich im Laufe der Zeit verändern, wobei sich rückläufiges Engagement einer Person mitunter durch vermehrtes Engagement anderer ausgleichen kann. In diesem Zusammenhang ist hervorzuheben, dass Aktivitäten oft nur von einer Person organisiert werden und dass Angebote bei schwindendem Interesse, privaten oder beruflichen Veränderungen somit abrupt wegfallen können. Hinsichtlich der von den Bürgermeister*innen genannten Herausforderungen der Bereitstellung eines adäquaten Kinderbetreuungsangebots sowie mobiler und stationärer Betreuung für ältere Menschen ist das Otelo-Konzept somit kein geeigneter Lösungsansatz, u. a. weil für diese Angebote Beständigkeit zentral ist.

\section{Schlussfolgerung}

Dem Spannungsfeld zwischen Ungezwungenheit und Ungebundenheit auf der einen Seite, und Zweckgebundenheit und Zielorientierung in Richtung räumliche Entwicklung auf der anderen Seite halten die Offenen Technologielabore insofern nicht stand, als strategisch ausgerichteten raumentwicklerischen Gestaltungsprozessen eine Analyse der lokalen bzw. regionalen Gegebenheiten vor- und eine Evaluierung der umgesetzten Maßnahmen nachgeschaltet ist. Zugunsten der Niederschwelligkeit der Offenen Technologielabore sind diese Komponenten im Otelo-Konzept bewusst nicht vorgesehen. Nichtsdestotrotz ist erkennbar, dass Otelo einen Beitrag zur Erweiterung des kommunalen Kultur- und Erholungsangebots und der Nutzung zur Ortskernbelebung und Standortaufwertung leistet. Darüber hinaus können in diesem Rahmen Initiativen gefördert werden, die einen Kontrapunkt zur Wachstumsorientierung setzen. Der Beitrag zur Kreativwirtschaft und zu kommerziell verwertbaren Innovationen - welcher speziell für kommunale Haushalte von Bedeutung wäre - bleibt vorerst überschaubar. Fest steht allerdings, dass die Beiträge der Offenen Technologielabore weniger in ländlichen Gemeinden, sondern überwiegend in kleinstädtischen, suburbanen und urbanen Gemeinden zu verorten sind. Wenngleich in Hinblick auf die Ergänzung der Angebote der Daseinsvorsorge eine Abstimmung der Themenzentrierung der Otelo-Gruppen mit den Agenden der Stadtentwicklung Sinn machen würde, widerspräche dies dem Otelo-Prinzip der Themenoffenheit. Dazu kommt, dass die befragten Bürgermeister*innen das Offene Technologielabor nicht als potenzielle Plattform zur Unterstützung der Gemeinde in der Koproduktion der Daseinsvorsorge sehen, sondern tendenziell als Plattform für soziokulturelle Initiativen.

Hinsichtlich der Einordnung von Otelo in das Konzept der Dritten Orte ist zu bemerken, dass Offenen Technologielabore trotz der Namens- und Themenverwandtheit zu Open Creative Labs, CreativeMornings und Co-Working-Spaces sich von diesen in einem wesentlichen Punkt unterscheiden: Otelos zeigen keine Bezüge zur Privatwirtschaft auf. Sie sind zivilgesellschaftlich ausgerichtet, was auch deren Potenziale und Limitationen hinsichtlich deren Bedeutung für die Koproduktion der Daseinsvorsorge erklärt.

Das Ziehen von Rückschlüssen, ob und inwiefern diese Einschätzung von den aktiven Otelo-Mitgliedern in den thematischen Gruppen (Nodes) geteilt wird bzw. diese sich im Laufe der Zeit verändert hat, ist auf Basis der vorliegenden empirischen Befunde, respektive des gewählten Studiendesigns nicht möglich: Erstens: Die Ausführungen stellen das Ergebnis der inhaltlichen Vertiefung einer wissenschaftlichen Abschlussarbeit dar („follow-up“), weshalb zwei unterschiedliche Datensets (2 verschiedene Zeitpunkte der Erhebung) miteinander verknüpft werden mussten. Zweitens: 
Im Zuge der zweiten Erhebung wurden die Perspektiven der Standortsprecher*innen und der Bürgermeister*innen der Standortgemeinden berücksichtigt. Drittens: Die Einschätzung der Autor*innen wurde nicht mit den Befragten reflektiert.

Die Erörterung der Perspektive von aktiven Otelo-Mitgliedern unter Berücksichtigung deren soziodemografischen und -ökonomischen Profils sowie deren Motivation zur Teilnahme an Otelo könnte Gegenstand weiterer Forschungen zur (aktuellen) Relevanz von Otelos als Dritte Orte im Kontext der kommunalen Entwicklung sein, ebenso die Auseinandersetzung der Frage, was die Attraktivität von Otelo und den persönlichen Mehrwert der Teilnahme im Vergleich zum Engagement in traditionellen Vereinen konkret bestimmt.

Diese Erkenntnisse wiederum könnten dazu genutzt werden, Entscheidungsmechanismen und räumliche Reichweiten Offener Technologielabore detaillierter nachvollziehen sowie die Konsequenzen der Themenoffenheit von Otelo und dessen Toleranz gegenüber verschiedenen Graden der Intensität der Teilhabe bewerten zu können.

Dies wiederum könnte in die Erörterung der Frage gipfeln, welche Rolle sogenannte Dritte Orte in der räumlichen Entwicklung generell spielen könnten, respektive spielen sollen.

Danksagung Die Autor*innen danken dem Gründer der Offenen Technologielabore sowie allen befragten Standortsprecher*innen und Bürgermeister*innen für ihre Bereitschaft zum Gespräch bzw. die Teilnahme an der schriftlichen Befragung und den Gutachter*innen für die intensive Auseinandersetzung unseres Beitrags.

Funding Die Publikationskosten wurden über den Open-AccessFonds der Universität für Bodenkultur Wien finanziert.

Funding Open access funding provided by University of Natural Resources and Life Sciences Vienna (BOKU).

Open Access Dieser Artikel wird unter der Creative Commons Namensnennung 4.0 International Lizenz veröffentlicht, welche die Nutzung, Vervielfältigung, Bearbeitung, Verbreitung und Wiedergabe in jeglichem Medium und Format erlaubt, sofern Sie den/die ursprünglichen Autor(en) und die Quelle ordnungsgemäß nennen, einen Link zur Creative Commons Lizenz beifügen und angeben, ob Änderungen vorgenommen wurden.

Die in diesem Artikel enthaltenen Bilder und sonstiges Drittmaterial unterliegen ebenfalls der genannten Creative Commons Lizenz, sofern sich aus der Abbildungslegende nichts anderes ergibt. Sofern das betreffende Material nicht unter der genannten Creative Commons Lizenz steht und die betreffende Handlung nicht nach gesetzlichen Vorschriften erlaubt ist, ist für die oben aufgeführten Weiterverwendungen des Materials die Einwilligung des jeweiligen Rechteinhabers einzuholen.
Weitere Details zur Lizenz entnehmen Sie bitte der Lizenzinformation auf http://creativecommons.org/licenses/by/4.0/deed.de.

\section{Literatur}

Bachinger K, Dörflinger A, Enichlmair C, Gavac K, Hölzl K, Koller W, Schneider H (2017) Siebenter Österreichischer Kreativwirtschaftsbericht. Schwerpunkt Crossover-Effekte und Innovation. Kreativwirtschaft Austria, Wien

Boyatzis RE (1998) Transforming qualitative information: thematic analysis and code development. SAGE, Thousand Oaks

Gläser J, Laudel G (2009) Experteninterviews und qualitative Inhaltsanalyse, 3. Aufl. VS, Wiesbaden

Growe A, Henn S, Scheffer C (2020) Co-Working-Spaces als Treffpunkte - Impulse für die Quartiersentwicklung? Das Beispiel Heidelberg. Standort 44:73-79. https://doi.org/10.1007/s00548-02000647-w

Heiler F (2008) Wie kommt das Neue ins Land? Nachhaltige Raumund Gesellschaftsentwicklung als Gegenstand der Emergenzund Innovationsforschung; Generierung profunder Innovation in ländlichen Kommunen. Dissertation, Universität für Bodenkultur Wien

Hollinetz M, Ottinger G, Hollinetz H, Schachinger R, Mader W, Hader A, Haider K (2012) Offenes Technologielabor Hand(lungs)buch. Das Handlungsbuch als Anregung und Einladung zum Handeln, Mitgestalten und Multiplizieren von Otelo. https://www.yumpu. com/de/document/read/11208041/handlungsbuch-Otelo. Zugegriffen: 6. Jan. 2021

Howaldt J, Schwarz M (2010) Soziale Innovation - Konzepte, Forschungsfelder und -perspektiven. In: Howaldt J, Jacobsen $\mathrm{H}$ (Hrsg) Soziale Innovation - Auf dem Weg zu einem postindustriellen Innovationsparadigma. VS, Wiesbaden, S 87-108

Müller P, Schneider N (2020) Wissensaustausch beim Frühstück "CreativeMornings" als Treffpunkt für Kultur- und Kreativschaffende in Berlin. Standort 44:80-85. https://doi.org/10.1007/ s00548-020-00644-z

Oldenburg R (1999) The great good place: cafés, coffee shops, bookstores, bars, hair salons, and other hangouts at the heart of a community. Marlowe, New York

Rogers E (2003) Diffusion of innovations, 4. Aufl. Free Press, New York

Schabasser H (2016) Offene Technologielabore als Beitrag zur Gemeinde- und Regionalentwicklung? Masterarbeit, Universität für Bodenkultur Wien

Schmidt S (2020) Open Creative Labs - Treffpunkte für Kreative? Standort 44:67-72. https://doi.org/10.1007/s00548-020-00638-x

Statistik Austria (2016) Urban-Rural-Typologie. https://www.statistik. at/web_de/klassifikationen/regionale_gliederungen/stadt_land/ index.html. Zugegriffen: 30. Apr. 2021

Waser D, Hollinetz H, Hufnagl K, Luger M, Hollinetz M, Mader W (2015) Otelo Wirkungsbericht 2011-2015. https://issuu.com/ otelo_offenes_technologielabor/docs/srs-bericht_otelo_highres. Zugegriffen: 27. Apr. 2021 (Otelo eGen)

Werlen B (2000) Sozialgeographie: eine Einführung. Paul Haupt, Bern, Stuttgart, Wien 